\title{
DIRECT NUMERICAL OPTIMISATION OF A FUEL JET PUMP BASED ON A PSEUDO-COMPRESSIBILITY METHOD USING CHARACTERISTIC BOUNDARY CONDITIONS
}

The paper tries to give an overview of the latest industrial development of the Department of Aircraft and Ships at the Budapest University of Technology and Economics.

The numerical investigation presented below based on Chorin's pseudo-compressibility method is used to make the incompressible Euler equations artificially hyperbolic [1]. A less dissipative flux difference splitting method is evolved to solve governing equations. A low cost 4th order Runge-Kutta method is applied for time marching in pseudo space. Both extrapolated and characteristic type boundary conditions are examined and compared with each other in qualitative accuracy and convergence point of view. Using the described computational tool a direct numerical optimisation of a fuel jet pump is performed.

\section{Notation}

Arabian

$A \quad$ Jacobian of flux $F$

$a_{i, j} \quad$ Element of $A$ matrix

$a \quad$ Pseudo sound speed

$c \quad$ Dumping coefficient

$e \quad$ Unit vector

$F \quad$ x com. of convective flux vec.

$G$ y com. of convective flux vec.

$\vec{H} \quad$ Total flux vector

$k \quad$ Stiffness coefficient

$L \quad$ Left eigenvector matrix

$\dot{m} \quad$ Mass flow

$N_{p} \quad$ Number of points in comp. domain

$N_{f} \quad$ Number of faces over the cell

$\vec{n} \quad$ Outside pointing unit normal vector

$P \quad$ Pseudo-compressibility parameter

$p \quad$ Static pressure

$t$ Time

$v \quad$ y direction velocity

$W \quad$ Vector of characteristic variables

$x, y, z$ Cartesian space

$U \quad$ Vector of conservative variables

$u \quad \mathrm{x}$ direction velocity

$\vec{V} \quad$ Velocity vector

$\dot{V} \quad$ Volume flow

Greek

$\alpha_{k} \quad$ Runge-Kutta coefficient

$\alpha \quad$ Damping coefficient

$\beta \quad$ Compressibility parameter

$\varepsilon \quad$ Volume flow rate
$\Gamma \quad$ Boundary of control surface

$\lambda_{i} \quad$ Eigenvalues of matrix A

$\tau \quad$ Weighting parameter

$\Omega \quad$ Control surface

\section{Numerical method}

\subsection{Governing equations}

The Euler equations can be derived from Newton's second law applied to a cubic fluid element only considering pressure forces and external forces. 2D incompressible Euler equations with the extension of the pseudo-incompressible term in dimensional form are given by [1]

$$
\frac{\partial U}{\partial t}+\frac{\partial F(U)}{\partial x}+\frac{\partial G(U)}{\partial y}=0
$$

where

$$
\begin{aligned}
& U=\left[P / \beta^{2}, u, v\right]^{T}, F(U)=\left[u, u^{2}+P, u v\right]^{T} \text { and } \\
& G(U)=\left[v, u v, v^{2}+P\right]^{T} .
\end{aligned}
$$

In this context $p$ is the static pressure, $\rho$ is the density, $u$ and $v$ is the Cartesian components of velocity vector, $\beta$ is the compressibility factor with the typical value of 3 and $P=p / \rho$. In order to satisfy consistency, when the steady state condition is reached, the first term of the continuity equation is vanished so the original form of the incompressible Euler equations is recovered. Introduction of total flux vector, $\vec{H}$, system (1) in compact form becomes

\footnotetext{
* Árpád Veress

Department of Aircraft and Ships, Faculty of Transportation, Department of Aircraft and Ships, Sztoczek u. 6 J. ép. 426 . H-1111, Budapest,

Hungary, tel. 00-36-1-463-1992, fax: 00-36-1-463-3080, e-mail: veress@rht.bme.hu
} 


$$
\frac{\partial U}{\partial t}+\vec{\nabla} \cdot \vec{H}(U)=0
$$

Integrating system (3) over a control volume $\Omega$, which is bounded by interface $\Gamma$ and applying the Gauss divergence theorem, one gets

$$
\frac{\partial}{\partial t} \iint_{\Omega} U d \Omega+\int_{\Gamma} \vec{H} \vec{n} d \Gamma=0
$$

where $\vec{n}=\left(n_{x}, n_{y}\right)$ is the local outside pointing unit vector and so

$$
H_{n}=\vec{H} \vec{n}=\left(\begin{array}{c}
V_{n} \beta^{2} \\
u V_{n}+P n_{x} \\
v V_{n}+P n_{y}
\end{array}\right),
$$

where $V_{n}\left(V_{n}=\vec{V} \vec{n}=\left(u \vec{e}_{x}+v \vec{e}_{y}\right)\left(n_{x} \vec{e}_{x}+n_{y} \vec{e}_{y}\right)=u n_{x}+v n_{y}\right)$ is the normal component of velocity vector.

\subsection{Discretization}

Concerning the system (4), in order to pass from continuous to a discrete form, a choice about the type of representation of the solution vector over the cell centered finite volume has to be made:

$$
U_{j}=\frac{1}{\Omega_{j}} \iint_{\Omega_{j}} U d \Omega
$$

By substituting (6) into the first integral in equation (4), and replacing the second integral by a summation over the number of faces $N_{f}$ of the chosen control volume $\Omega_{j}$, equation (4) can be written in the following semi-discrete form for the point $j$ :

$$
\frac{\partial}{\partial t} U_{j}=-\frac{1}{\Omega_{j}} \sum_{k=1}^{N_{j}}\left[H_{n}\right]_{j, k} \Gamma_{j, k} .
$$

For a higher order reconstruction $\kappa$-class of a higher order scheme approach is used for determining the cell face flux function using flow variables at left and right states [2].

In case of spatial discretisation, flux difference splitting methods have the lowest level of indispensable dissipation compared with the other type of splitting or artificial dissipation methods. This feature is also important in the extension of the code for accurate modeling of shear layers in viscous flow. Hence, the total flux functions (5) at the interface of two adjacent cells are written in following form

$$
H_{n}=\frac{1}{2}\left(H_{n, i}+H_{n, i+1}\right)+\frac{1}{2}\left(\hat{R} \hat{\Lambda}^{-} \hat{L}+\hat{R} \hat{\Lambda}^{+} \hat{L}\right) \Delta U,
$$

where $\hat{R}, \hat{L}=\hat{R}^{-1}$ are the right and left eigenvector-matrixes composed by an average of right an left states:

$$
\hat{R}=\left(\begin{array}{ccc}
0 & \beta^{2} a & -\beta^{2} a \\
-n_{y} & \beta^{2} n_{x}+u\left(V_{n}+a\right) & \beta^{2} n_{x}+u\left(V_{n}-a\right) \\
n_{x} & \beta^{2} n_{y}+v\left(V_{n}+a\right) & \beta^{2} n_{y}+v\left(V_{n}+a\right)
\end{array}\right),
$$

where $a=\sqrt{V_{n}^{2}+\beta^{2}}$ is the pseudo sound speed and $\Lambda^{ \pm}$are the positive and negative eigenvalue-matrixes respectively.
For marching in pseudo time the computationally inexpensive fourth order Runge-Kutta method is used to solve equation (7):

$$
\begin{gathered}
U^{0}=U^{n} \\
U^{k}=U^{0}+\alpha_{k} \Delta t \Re\left(U^{k-1}\right) \quad k=1, \ldots 4 \text { and } \\
U^{n+1}=U^{m} \\
\alpha_{k}=\frac{1}{k_{\text {max }}+1-k} .
\end{gathered}
$$

\subsection{Boundary conditions}

The governing equations are of hyperbolic type, so the number of physical and numerical boundary conditions is determined by the theory of characteristics (inlet: $p_{m}^{t o}, \alpha_{i n}$, outlet: $p_{\text {out }}^{\text {st }}$, and mirror solid wall). The numerical boundary conditions are computed by both linear extrapolation and characteristic type manner and they are compared with each other by means of qualitative accuracy and number of iteration.

In case of linear extrapolation the unknown numerical flow variables are determined by a simple linear extrapolation.

The system of Euler equations is the hyperbolic type and it can be described by the wave like behaviour, the information propagates along the characteristics. The slope of these characteristic curves is determined by the eigenvalues of Jacobian, while the unknowns are written in the expression of characteristic variables, which are constants along their characteristic lines, along which the information propagates. Take the theory of characteristic into consideration; more consistent boundary treatment is available for determination of numerical boundary conditions expecting less number of iteration.

There is one outgoing characteristic at the inlet, which means that

$$
\begin{aligned}
& W_{n}^{(3)}=\left(V_{n}+a\right)_{I} p_{I}-\beta^{2} V_{n, I}= \\
& =\left(V_{n}+a\right)_{B=\lim _{t \rightarrow \infty} I} p_{B}-\beta^{2} V_{n, B}
\end{aligned}
$$

relation should keep over the boundary per iterations ( $I$ : inner parameters, $B$ : boundary values). Using equation:

$$
V_{n}=V\left(\frac{-n_{x}-n_{y} \operatorname{tg} \alpha}{\sqrt{1+\operatorname{tg}^{2} \alpha}}\right)
$$

( $\alpha$ is the flow angle, $V$ is the length of velocity vector) equation (11) leads to a second degree equation for $V_{B}$ to solve. Finally all other numerical unknowns are easy to be recovered.

There are two ingoing chraracteristics at the otlet, which means that next charactersitic variables should be kept constant:

$$
\begin{aligned}
& W_{n}^{(1)}=V_{t, I}=V_{t, B}, W_{n}^{(2)}=\left(V_{n}-a\right)_{I} p_{I}-\beta^{2} V_{n, I}= \\
& =\left(V_{n}-a\right)_{B=\lim _{t \rightarrow \infty} I} p_{B}-\beta^{2} V_{n, B},
\end{aligned}
$$


where $V_{t}$ is the covariant velocity. Equations (13) contain only two unknowns, namely $V_{t, B}$ and $V_{n, B}$ to determine explicitly. Finally the components of velocity vector can easily be calculated.

\subsection{Validation}

Circular bump in 2D channel test case is used for validation [3]. The results of own code and FLUENT commercial software

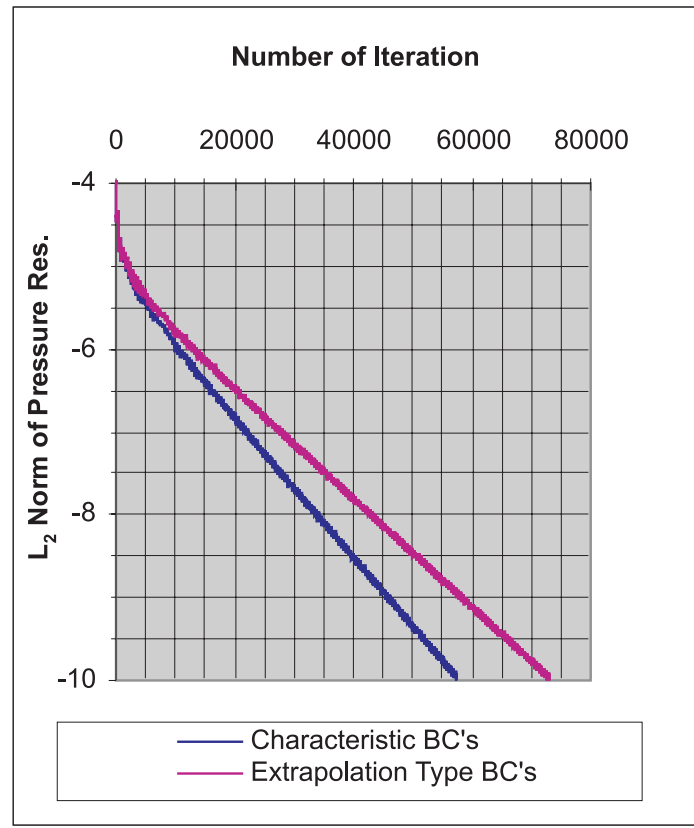

Fig. 1. Comparison of convergence history by means of extrapolation and characteristic types boundary conditions

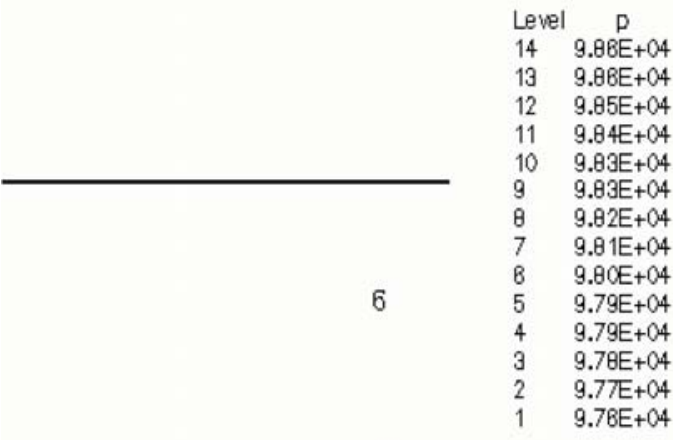

5

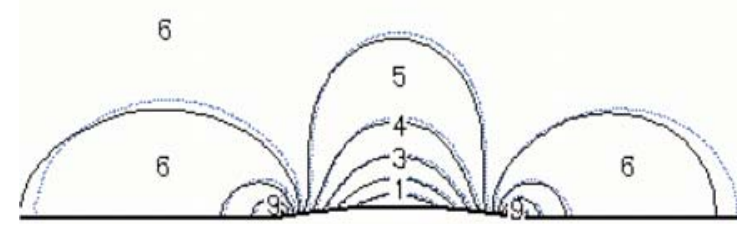

Fig. 2. Comparison of pressure distribution: solid line: FLUENT commercial code, dotted line: own code are compared with each other at the same geometry, initial and physical boundary conditions. The difference between two results can be found in Fig. 2 and it can be concluded that the difference is negligible. The pressure distributions with the application of two different boundary conditions cover each almost exactly. The characteristic type inlet and outlet boundary condition shows significant improvements (Fig. 1) concerning the number of iteration and so reduces computational time compared to the extrapolation type one. The convergence rate is characterised by the normalized $L_{2}$ norm of pressure residual:

$$
\left\|\frac{\Delta p}{p}\right\|=\log _{10} \sqrt{\frac{1}{N_{p}} \sum_{i=1}^{N_{p}}\left(\frac{\Delta p_{t}}{p_{t}}\right)^{2}} .
$$

All the numerical computations were performed at the Center of Information Systems (CIS) of the Budapest University of Technology and Economics on a supercomputer. This serverfarm contains four Compaq 4100 nodes: 16 x EV5.6 (21164A, $600 \mathrm{Mhz}, 8$ MByte cache) Alpha CPU, 32 GByte memory, 0.62 TByte harddisc.

\section{2D Direct numerical optimisation of a fuel jet pump}

With the application of the code presented in the previous sub-chapters, a new design guideline is developed for the optimisation of a fuel jet pump. Nowadays, this device is widely used in the internal fuel system of a car fuel tank system. The jet pump deliveries fuel from one part of the tank to the other. During the operation, the most important, apart from guaranteeing the maximum volumetric flow rate, is the reliability which can be reached by the optimal design of mixture area.

Concerning the 2D analysis, a low-pressure zone is appeared locally in the outside part of the right side return bend resulting swirling flows (Fig. 3/A). The origin of this phenomenon can be attributed to two occurrences. The most meaningful is the concave curvature effect.

$$
\rho\left(\frac{V_{t}^{2}}{R_{c}}\right)=\frac{\partial p}{\partial n}
$$

Near the wall, to ensure radial force balance - kept by centrifugal force and pressure (15) - the absolute velocity decreases. On the other hand, at the inlet of the diffuser the contraction is high because of a sharp corner. Near the inlet of return bend, when the effect of contraction is diminishing and the flow is spread over, the absolute velocity is also decreasing. This phenomenon is superposed to the velocity decrement caused by concave curvature effect results so intensive adverse pressure gradient increment that the stream is not able to follow the solid surface any more and swirling flow is evolved at the outer, concave part of return bend (Fig. 3/A)

According to engineering and computational investigation, the chamfered throat at the inlet of the jet diffuser helps to avoid swirling flow (Fig. 3/B). Furthermore, the flow transportation is also improved by using a relatively small flow driver lug located at the orifice outlet of the jet pump (Fig. 4). 

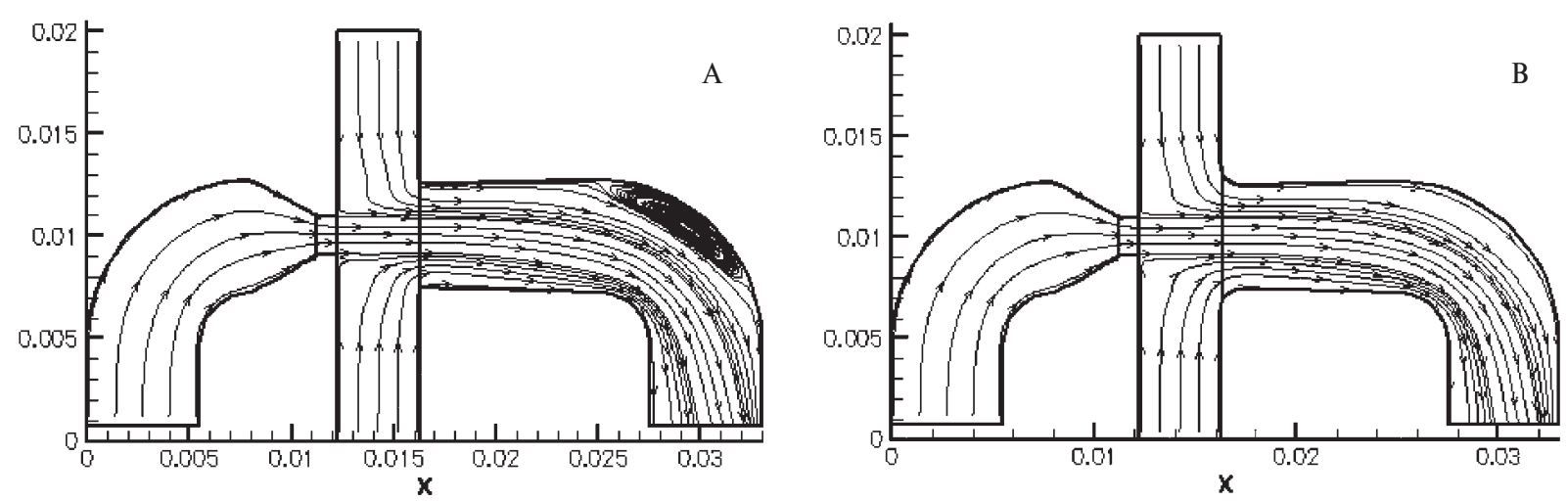

Fig. 3. Stream-traces in 1.9 mm orifice diameter fuel jet pump. A: without (basic configuration), B: with chamfered throat
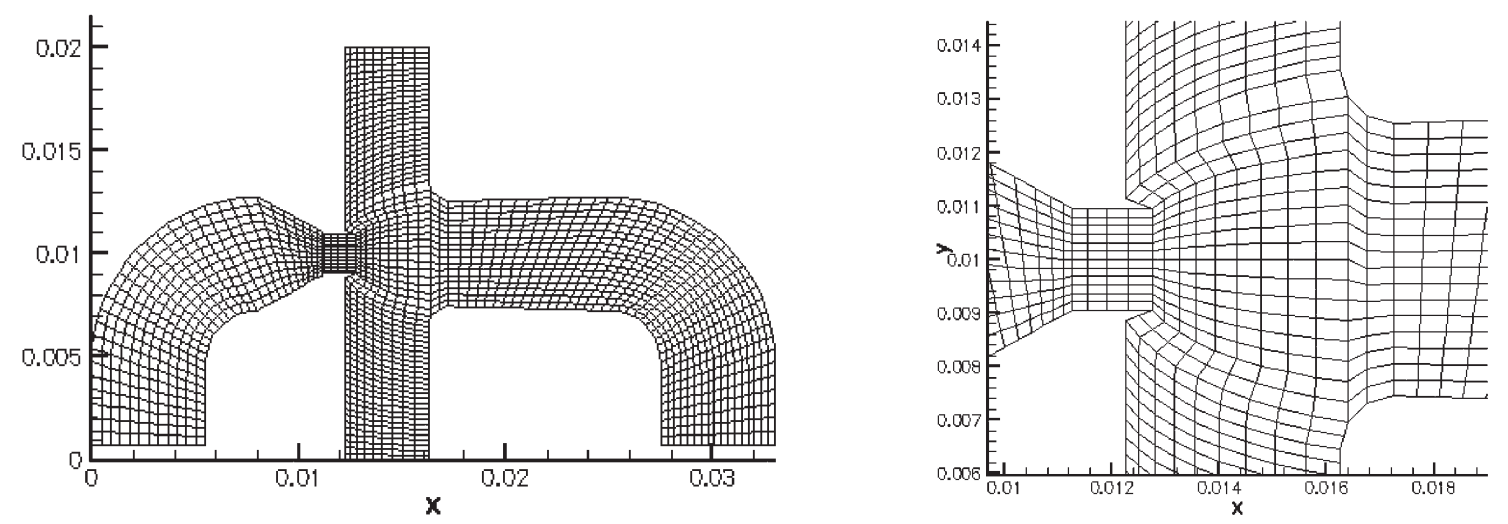

Fig. 4. Numerical model of $1.9 \mathrm{~mm}$ orifice diameter fuel jet pump with structured grid applying chamfered throat ${ }^{+}$and $0.5 \mathrm{~mm}$ long flow driven *

Finally - keeping the smallest modification concerning the production cost - the combination of three main geometrical sizes has been investigated, namely; the inlet width (inlet for transported fuel), the diffuser inlet diameter and the diffuser length, which are changed over the causality distance by 5 equivalent size-steps and examined with each other. They might have an optimal set of configuration, which belongs to the maximum outlet flow. This direct numerical simulation means $5^{3}$ 2D computations, which takes at about 12-24 hours in the function of grid fineness and the specifications of PC. After making optimisation, the results justify our expectations, as it can be seen in Table 1:
The optimum model is called Optimised Geometry with the maximum flow transportation of 3.18. It is interesting also to analyse this configuration. It can be observed in Fig. 5/B that orifice flow gives more rate of kinetic energy to the transported flow than as it can be observed in Fig. 5/A. The streamline distribution becomes more regular, so the risk for swirling flows is decreased and more mass is transported. The fluid dynamics phenomena caused by curvature effects in the return bend have significant effect on processes in the central part of the pump. A low-pressure region is developed at the convex curvature of return bend. Hence, more mass can come from the lower inlet section

Results of optimisation procedure in the function of volume flow rate

Table 1

\begin{tabular}{|c|c|c|c|c|c|}
\hline Models & Basic Configuration & $\begin{array}{c}\text { Basic }+ \text { Chamfered } \\
\text { Throat }\end{array}$ & $\begin{array}{c}\text { Basic }+ \text { Chamfered } \\
\text { Throat }+0.2 \mathrm{~mm} \\
\text { Flow Driven Lug }\end{array}$ & $\begin{array}{c}\text { Basic+Chamfered } \\
\text { Throat }+0.5 \mathrm{~mm} \\
\text { Flow Driven Lug }\end{array}$ & Optimised Geometry \\
\hline$\dot{V}_{\text {out }}^{\text {sum }}[1 / \mathrm{h}]$ & 307.0 & 330.69 & 335.08 & 338.465 & 369.1 \\
\hline$\dot{V}_{\text {in }}^{\text {sum } p l y}[1 / \mathrm{h}]$ & 129.8 & 128.83 & 127.73 & 128.45 & 115.9 \\
\hline$\varepsilon=\frac{\dot{V}_{\text {out }}^{\text {sum }}}{\dot{V}_{\text {in }}^{\text {sum } p l y}}$ & 2.36 & 2.57 & 2.62 & 2.63 & 3.18 \\
\hline
\end{tabular}



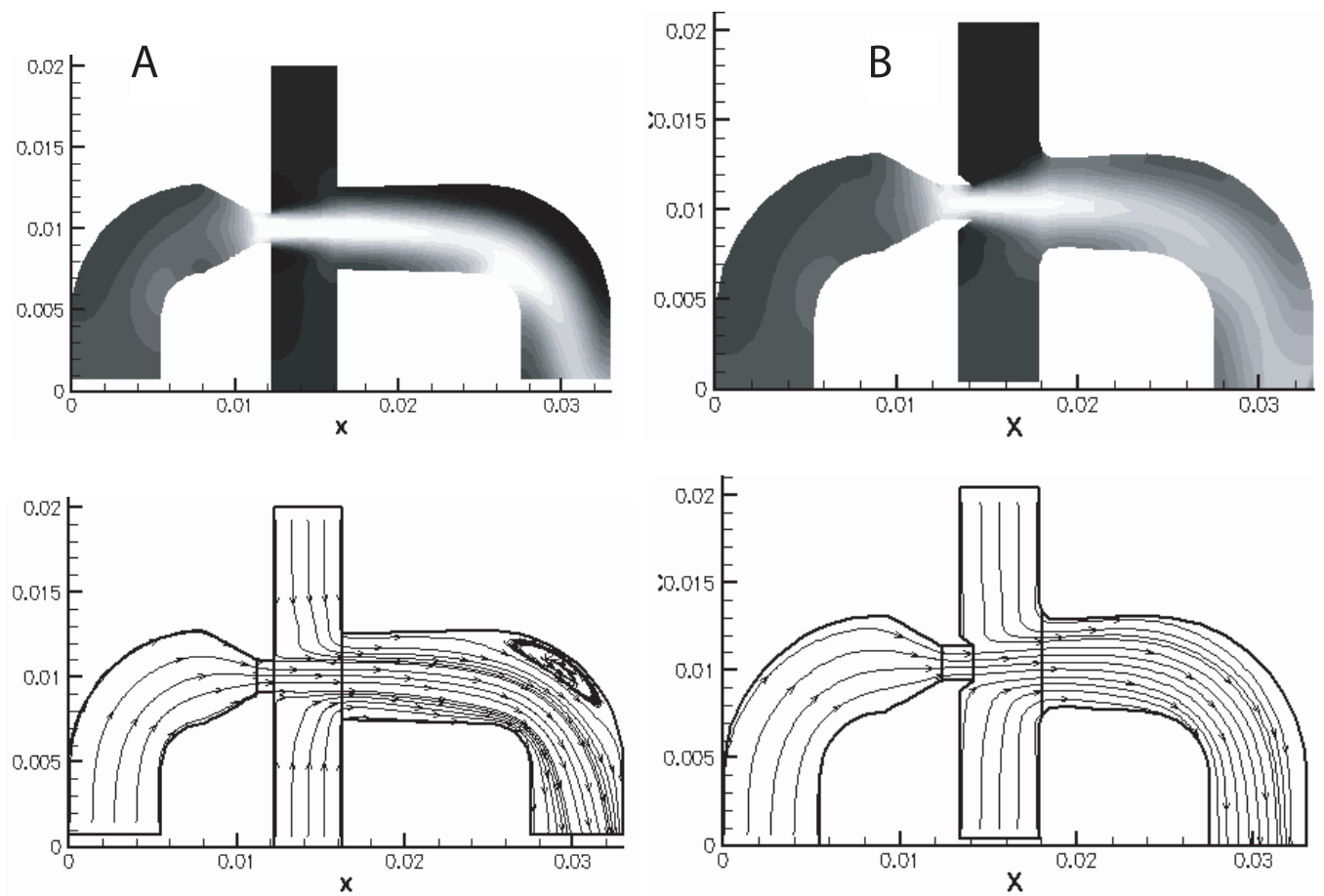

Fig. 5. Velocity distribution $[\mathrm{m} / \mathrm{s}]$ (above) and stream-traces (below) in the $1.9 \mathrm{~mm}$ orifice diameter jet pump.

A: Basic configuration B: Optimised geometry

into the diffuser, and so, the stream, which comes from the orifice, is not straight (horizontally) any more but rather slightly turns up. This flow turning makes the streamline distribution more uniform and has a strong effect to sweep out the swirling flow from the bend.

\section{Summary and conclusions}

Installation of 2D Pseudo-compressibility method is presented for modelling ideal incompressible flow by solving Euler equations to numerically investigate and optimise a small-size fuel jet pump. 4th order Runge-Kutta method is applied for time marching, while the flux difference splitting technique with -class of a higher order scheme approach is used for spatial discretisation. Extrapolation and characteristic type boundary conditions are examined by means of qualitative accuracy and convergence manner. It can be concluded that the scheme using more consistent characteristic boundary conditions shows faster convergence properties to reach steady state conditions saving considerable computational time. After validation of the code a certain combination of the pump geometry is defined, which has the highest effect on flow transportations. Physical interpretation of fluid dynamics phenomena is presented in the all examined cases besides determining an optimum size configuration.

\section{References}

[1] CHORIN, A. J.: A Numerical Method for Solving Incompressible Viscous Flow Problems. Journal of Computational Physics, 2, 12-26, 1967.

[2] MANNA, M.: A Three Dimensional High Resolution Compressible Flow Solver. PhD Thesis in Catholic University of Louvain, 1992.

[3] VERESS, Á.: Numerical Methods and Applications for Flow Calculations in Turbo and Fluid Machines. Ph. D. thesis, Department of Aircraft and Ships, Budapest University of Technology and Economics, 2004. 